\title{
Reversible dentate nucleus and corpus callosum lesions in acute Legionnaires' Disease
}

\author{
Karan Topiwala, Karan Tarasaria, Avinash Prasad
}

\section{CASE REPORT}

A 41-year-old man presented with a two-day history of fever, malaise and diarrhoea. He was disoriented to time and situation with mild dysarthria on neurologic examination. He was found to be hyponatremic (132 $\mathrm{mmol} / \mathrm{L}$ ) and a chest X-ray showed left lower lobe consolidation. He was started on broad-spectrum antibiotics including two doses of Metronidazole. MRI of the brain without contrast showed T2/FLAIR hyper intensities within bilateral DN and in the central aspect of the splenium of the corpus callosum (CC) (Figure 1). His urine Legionella Pneumophila serogroup-1 antigens came back positive and antibiotics were de-escalated to Levofloxacin for two weeks. On his five-week posthospitalization follow-up, he was noted to have persistent dysarthria for which he continued to work with speech therapy twice a week. Repeat MRI brain with and without contrast six weeks from his initial MRI scan showed a complete resolution of the previously noted abnormal signal hyperintensity within the dentate nuclei and the corpus callosum. He was subsequently seen in clinic ten weeks from discharge and was noted to be asymptomatic.

\section{DISCUSSION}

The dentate nucleus is the largest and most lateral of the four deep cerebellar nuclei, placed directly adjacent to the vermis and the roof of the fourth

Karan Topiwala ${ }^{1}$, Karan Tarasaria ${ }^{1}$, Avinash Prasad ${ }^{1}$

Affiliation: 'University of Connecticut School of Medicine, Hartford Hospital, Department of Neurology, Seymour St., Hartford, CT, USA.

Corresponding Author: Karan Topiwala, University of Connecticut School of Medicine, Hartford Hospital, Department of Neurology, 80 Seymour St., Hartford, CT 06106, USA; Email: topiwala@uchc.edu

Received: 12 May 2018

Accepted: 19 June 2018

Published: 31 July 2018

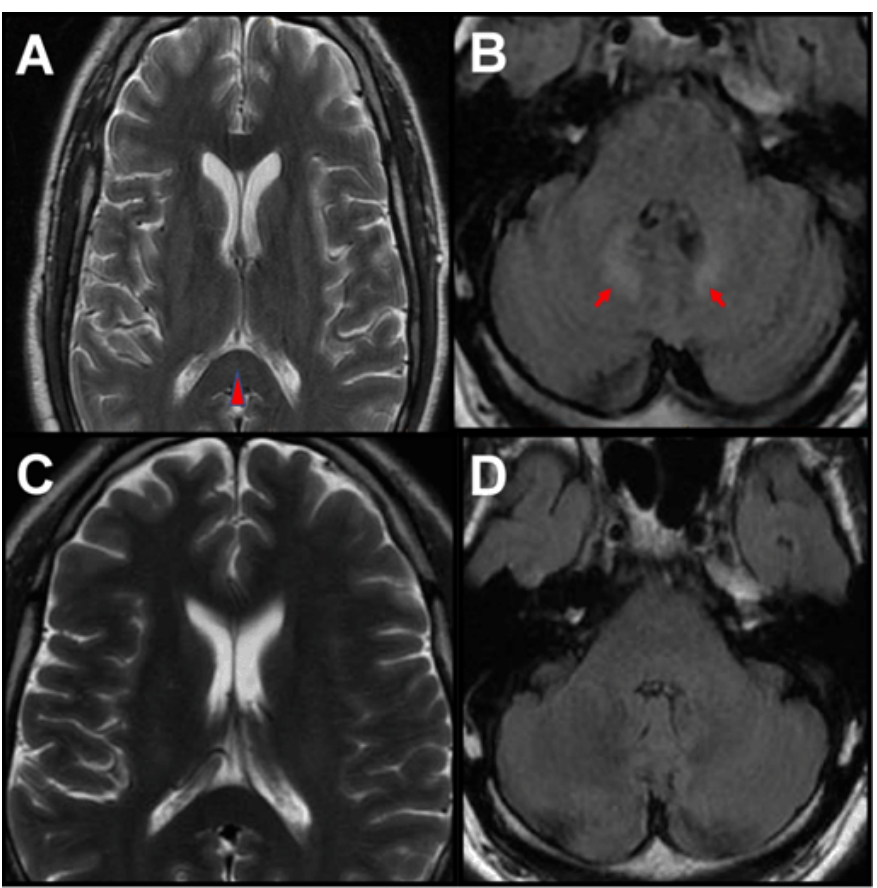

Figure 1: MRI Brain without contrast; MRI brain showing T2hyperintesnity (A) in the center of the splenium of the corpus callosum and FLAIR hyperintensity of bilateral dentate nuclei (B) with interval resolution (C, D) on follow-up imaging performed seven weeks later.

ventricle bilaterally. It receives ascending proprioceptive projections of the spinocerebellar tracts via the inferior cerebellar peduncles, as well as descending projections from the motor cortex. Efferent pathways comprise of the dentato-rubral outflow to the contralateral ventro-lateral thalamus [1]. Neuroimaging abnormalities of the dentate nuclei have a broad differential diagnosis. Bilateral T2weighted-MRI hyperintensities of the Dentate Nucleus (DN) are seen with metabolic disorders (maple syrup urine disease, canavan's disease and glutaric aciduriatype-1) and metronidazole toxicity [1]. Since the first case report of Metronidazole induced reversible DN/CClesions in 1995, all subsequent cases have documented at least a four-week exposure to the antibiotic with or without supra-therapeutic levels, making it an unlikely cause of our patient's presentation [2]. Inherited metabolic 


\section{EDORIUM Journals}

disorders were unlikely since they typically present in infancy and are rapidly fatal when left untreated. On the other hand, T1 hyperintensity of the bilateral DN is suggestive of blood products (hypertensive bleeds) or mineralization (Iron deposition seen in multiple sclerosis and neurodegenerative conditions or Calcium deposition characteristic of Fahr's disease). However, neither did our patient have T1 signal change on his initial MRI, he also did not have any corresponding succeptibility artifact on his gradient ECHO sequence that would suggest the presence of blood or calcium. While gadolinium deposition can produce T1 signal change in the dentate nuclei, our patient had had fewer than three gadoliniumenhanced imaging studies prior to this presentation making it an unlikely etiology. $40-50 \%$ of patients with acute Legionnaires' Disease (LD) can develop neurologic symptoms such as ataxia, dysarthria and confusion [3]. While most have normal MRI findings, functional imaging with single photon emission tomography (SPECT) has often shown cerebellar perfusion abnormalities [4]. Two of seven patients with LD described by Johnson et al. had abnormal findings on head CT scan, one showed cerebral edema while the other had multifocal lesions with subsequent development of necrotizing hemorrhagic leucoencephalitis [5]. Spieker et al [6] and Sommer et al. [7] described diffuse subcortical white matter changes consistent with Acute Disseminated Meningoencephalittis (ADEM). Two patients who presented with seizures following acute LD were found to have bilateral mesial temporal FLAIR hyper intensities [8]. A 44-year-old man with Legionella bozemanii pneumonia was found to have bilaterally symmetrical foci of demyelination in the brainstem [9]. Reversible lesions of the posterior corpus callosum have been described with a variety of disorders such as Marchiafava-Bingnami disease, haemolyticuraemic syndrome, rotavirus infection, acute cerebellitis and High altitude cerebral oedema (HACE) [10]. These patients often present with ataxia and dysarthria, which is hypothesized to result from cerebellar diaschisis [3]. Similar to these case reports, our patient demonstrated reversible splenial T2-hyperintensities with spontaneous clinico-radiological resolution over the next eight weeks. However, DN involvement in acute LD has rarely been reported. The exact pathophysiology of such injury remains elusive, with neuropathologic evaluation showing anoxic injury with rare instances of direct gram-negative bacterial invasion [11]. Legionella is also known to produce cytotoxins that can generate cellmembrane pores in eukaryotic cells resulting in cell death from osmotic lysis, which may explain the subsequent development of cytotoxic edema [10]. Most patients with neurologic symptoms in acute LD improve spontaneously with resolution of the lung disease and do no need any form of pharmacologic therapy [10].

\section{CONCLUSION}

LD is commonly associated with cerebellar symptoms and dysarthria. These are hypothesized to result from cerebellar diaschisis resulting from reversible DN and CC lesions seen infrequently on MRI. The symptoms are typically reversible without any treatment. While anoxic injury with rare direct bacterial invasion of the DN has been noted at autopsy, bilateral dentate nuclei lesions on MRI in acute LD, is an uncommonly reported finding.

\section{REFERENCES}

1. Bond KM, Brinjikji W, Eckel LJ, Kallmes DF, McDonald RJ, Carr CM. Dentate update: Imaging features of entities that affect the dentate nucleus. AJNR Am J Neuroradiol 2017 Aug;38(8):1467-74.

2. Woodruff BK, Wijdicks EF, Marshall WF. Reversible metronidazole-induced lesions of the cerebellar dentate nuclei. N Engl J Med 2002 Jan 3;346(1):689.

3. Shetty KR, Cilyo CL, Starr BD, Harter DH. Legionnaires' disease with profound cerebellar involvement. Arch Neurol 1980 Jun;37(6):379-80.

4. Daaboul Y, Vern BA, Blend MJ. Brain SPECT imaging and treatment with IVIg in acute post-infectious cerebellar ataxia: Case report. Neurol Res 1998 Jan;20(1):85-8.

5. Johnson JD, Raff MJ, Van Arsdall JA. Neurologic manifestations of Legionnaires' disease. Medicine (Baltimore) 1984 Sep;63(5):303-10.

6. Spieker S, Petersen D, Rolfs A, et al. Acute disseminated encephalomyelitis following Pontiac fever. Eur Neurol 1998 Oct;40(3):169-72.

7. Sommer JB, Erbguth FJ, Neundörfer B. Acute disseminated encephalomyelitis following Legionella pneumophila infection. Eur Neurol 2000;44(3):1824.

8. Karim A, Ahmed S, Rossoff LJ. Legionnaire's disease associated with acute encephalitis and arrhythmia. Crit Care Med 2002 May;30(5):1028-9.

9. Platzeck C, Foerster EC, Schneider MU, et al. Encephalitis in Legionella bozemanii pneumonia. [Article in German]. Dtsch Med Wochenschr 1990 Dec 21;115(51-52):1956-9.

10. Morgan JC, Cavaliere R, Juel VC. Reversible corpus callosum lesion in legionnaires' disease. J Neurol Neurosurg Psychiatry 2004 Apr;75(4):651-4.

11. Pendlebury WW, Perl DP, Winn WC Jr, McQuillen JB. Neuropathologic evaluation of 40 confirmed cases of Legionella pneumonia. Neurology 1983 Oct;33(10):1340-4.

$* * * * * * * * *$

Keywords: Corpus callosum, Dentate nucleus, Dysarthria, Legionnaires' disease 


\section{How to cite this article}

Topiwala K, Tarasaria K, Prasad A. Reversible dentate nucleus and corpus callosum lesions in acute Legionnaires' Disease. Int J Case Rep Images 2018;9:100943Zo1KT2018.

Article ID: 100943Z01KT2018

$* * * * * * * * *$

doi: 10.5348/100943Zo1KT2018CL

$* * * * * * * * *$

\section{Author Contributions}

Karan Topiwala - Substantial contributions to conception and design, Acquisition of data, Drafting the article, Revising it critically for important intellectual content, Final approval of the version to be published

Karan Tarasaria - Substantial contributions to conception and design, Drafting the article, Final approval of the version to be published

Avinash Prasad - Substantial contributions to conception and design, Drafting and reviewing the article, Final approval of the version to be published

\section{Guarantor of Submission}

The corresponding author is the guarantor of submission.

\section{Source of Support}

None.

\section{Consent Statement}

Written informed consent was obtained from the patient for publication of this clinical image.

\section{Consent Statement}

Written informed consent was obtained from the patient for publication of this clinical image.

\section{Conflict of Interest}

Authors declare no conflict of interest.

\section{Copyright}

(C) 2018 Karan Topiwala et al. This article is distributed under the terms of Creative Commons Attribution License which permits unrestricted use, distribution and reproduction in any medium provided the original author(s) and original publisher are properly credited. Please see the copyright policy on the journal website for more information.
Access full text article on other devices

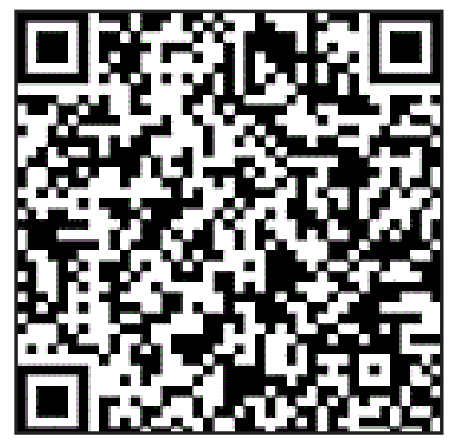

Access PDF of article on other devices

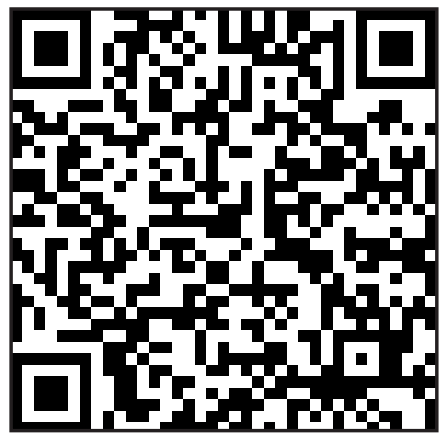

